\title{
Far-infrared Excesses in Classical Be Stars
}

\author{
A.S. Miroshnichenko, K.S. Bjorkman \\ Ritter Observatory, University of Toledo, Dept. of Physics and \\ Astronomy, Toledo, $\mathrm{OH} 43606, \mathrm{USA}$
}

\begin{abstract}
A sample of Be stars with large far-infrared excesses detected by IRAS is selected and their high-resolution IRAS images are examined. The far-infrared emission from most of them is marginally extended and is associated with the optical sources. Modeling of the stars' spectral energy distributions allows us to rule out the infrared cirrus nature of the excesses. We suggest that the excesses are more likely due to circumstellar dust, possibly formed early in the evolution of the star.
\end{abstract}

\section{Introduction}

Classical Be stars are intermediate-mass non-supergiant rapidly rotating emissionline stars which are believed to be surrounded by geometrically thin gaseous disks. Coté \& Waters (1986) studied far-IR excesses in these stars using the IRAS Point Source Catalog (PSC) and attributed them to free-free and freebound radiation of circumstellar gas. Gaustad \& Van Buren (1993) analysed low-resolution IRAS maps around bright stars with extended $60 \mu \mathrm{m}$ emission (including a number of Be stars) and suggested that the latter is due to interstellar dust emission (infrared cirrus). However, as Be stars are main-sequence objects, they might retain a certain amount of protostellar dust, which is found around their precursors, Herbig Ae/Be stars, and their lower-mass main-sequence counterparts, $\beta$ Pictoris (Vega-type) stars. Note that other emission-line B-type stars surrounded by circumstellar dust exist (LBVs, post-AGB stars, planetary nebulae). Recently Miroshnichenko et al. (1999) discovered emission in the $\mathrm{H} \alpha$ line in two late B-type stars with large IR-excesses, HD 4881 and HD 5839. Their location in the HR diagram and shape of the far-IR excesses led us to a suggestion that these are classical Be stars surrounded by distant, but still circumstellar, dust. The purpose of this project is to search for traces of circumstellar dust in the far-IR excesses of other Be stars.

\section{Source selection}

In order to select candidates for such a search we cross-correlated the Be star catalog (Jaschek \& Egret 1982) and the IRAS PSC and FSC (Faint Source Catalog) and found nearly $300 \mathrm{Be}$ stars, which are located within the IRAS positional error boxes. From this list we extracted the objects with IR fluxes not consistent with those expected from free-free or photospheric radiation, i.e. very slowly falling or rising towards longer wavelengths. High-resolution IRAS 
(HIRES) images of $1^{\circ} \times 1^{\circ}$ areas around our objects' positions were requested from the Infrared Processing and Analysis Center in Pasadena, California (IPAC). The main questions we address in our analysis of the IR images and colors are as follows: are the infrared sources point-like or extended and is the infrared emission due to circumstellar or interstellar dust?

Table 1. Be stars with unusual IR excesses

\begin{tabular}{|c|c|c|c|c|c|c|c|c|}
\hline $\mathrm{HD}$ & $V$ & Sp.T. & IRAS & [60] & Off. & $D(\mathrm{pc})$ & $\log \mathrm{L} / \mathrm{L}_{\odot}$ & Dust \\
\hline 4881 & 6.23 & B9.5 IV & $00487+5118$ & 11.2 & 28.0 & $350_{-70}^{+110}$ & $2.8 \pm 0.3$ & $\mathrm{Y}$ \\
\hline 5839 & 6.67 & B9 IV & $00581+6905$ & 3.72 & 3.4 & $360_{-100}^{+20}$ & $2.6 \pm 0.4$ & $\mathrm{Y}$ \\
\hline 6343 & 7.27 & $\mathrm{~B} 7 \mathrm{~V}$ & $01026+6541$ & 2.42 & 13.7 & $252_{-40}^{+60}$ & $2.3 \pm 0.2$ & $\mathrm{Y}$ \\
\hline 9709 & 7.07 & B8 $v$ & $F 01330+4651$ & 0.60 & 4.2 & $295_{-60}^{+100}$ & $2.3 \pm 0.2$ & $\mathrm{Y}$ \\
\hline 22298 & 7.60 & $\mathrm{~B} 2 \mathrm{~V}$ & $03341+5500$ & 1.28 & 5.1 & $360_{-85}^{+165}$ & $3.6 \pm 0.4$ & $\mathrm{~N}$ \\
\hline 26398 & 6.96 & B7 III & $04082+1631$ & 1.61 & 7.6 & $\geq 335$ & $\geq 3.0$ & $\mathrm{Y}$ \\
\hline 32343 & 5.08 & $\mathrm{~B} 3 \mathrm{v}$ & $05017+5854$ & 2.24 & 10.9 & $205_{-28}^{+38}$ & $3.4 \pm 0.1$ & $\mathrm{~N}$ \\
\hline 32991 & 5.78 & $\mathrm{~B} 2 \mathrm{v}$ & $05049+2138$ & 3.39 & 11.2 & $315_{-65}^{+110}$ & $3.9 \pm 0.3$ & $\mathrm{~N}$ \\
\hline 35347 & 8.92 & B1: IV: & $05221+2934$ & 0.93 & 29.9 & & & $\mathrm{~N}$ \\
\hline 36576 & 5.69 & B2 IV-V & $05306+1830$ & 1.30 & 14.1 & $\geq 570$ & $\geq 4.2$ & $\mathrm{~N}$ \\
\hline 37318 & 8.39 & B1 v & $05358+2826$ & 2.75 & 14.8 & & & $\mathrm{Y}$ \\
\hline 38120 & 9.10 & B9 $\mathrm{v}$ & $05407-0501$ & 15.0 & 27.1 & $\geq 420$ & $\geq 2.1$ & $\mathrm{Y}$ \\
\hline 251726 & 9.38 & B1 v: & $06039+1902$ & 16.2 & 14.1 & & & $\mathrm{Y}$ \\
\hline 50083 & 6.92 & $\mathrm{~B} 2 \mathrm{IV}$ & $06491+0508$ & 3.61 & 12.1 & & & $\mathrm{Y}$ \\
\hline 55439 & 8.47 & $\mathrm{~B} 2 \mathrm{v}$ & $07101-0945$ & 1.77 & 35.7 & 1000 & $3.7 \pm 0.2$ & $\mathrm{Y}$ \\
\hline 68980 & 4.78 & B1.5 III & $08115-3544$ & 1.03 & 3.8 & $300_{-40}^{+50}$ & $4.2 \pm 0.2$ & $\mathrm{~N}$ \\
\hline 124771 & 5.06 & B4 v & F14162-7952 & 8.56 & 10.0 & $170 \pm 15$ & $3.1 \pm 0.1$ & $N$ \\
\hline 131492 & 5.11 & $\mathrm{~B} 4 \mathrm{~V}$ & $14526-6234$ & 1.19 & 29.1 & $256_{-36}^{+52}$ & $3.5 \pm 0.2$ & $\mathrm{~N}$ \\
\hline 147756 & 8.60 & $\mathrm{~B} 1.5 \mathrm{~V}$ & $16221-4525$ & 4.06 & 8.4 & & & $Y$ \\
\hline 174571 & 8.89 & B3 v: & $18483+0838$ & 1.83 & 15.1 & 1050 & 3.9 & $\mathrm{Y}$ \\
\hline 225985 & 9.08 & B1 v: & $19476+3249$ & 1.63 & 11.5 & & & $\mathrm{Y}$ \\
\hline 203467 & 5.18 & B3 III & $21183+6439$ & 14.5 & 16.0 & $330_{-45}^{+65}$ & $3.8 \pm 0.2$ & $\mathrm{~N}$ \\
\hline 204722 & 7.67 & $\mathrm{~B} 2 \mathrm{v}$ : & $21273+4407$ & 2.56 & 7.2 & $510_{-125}^{+250}$ & $3.3 \pm 0.4$ & $Y$ \\
\hline 240010 & 9.48 & B1: IV: & $22365+5534$ & 5.54 & 6.5 & $\geq 470^{-120}$ & & $\mathrm{Y}$ \\
\hline
\end{tabular}

In column 4 "F" designates FSC objects, fluxes at $60 \mu \mathrm{m}$ are given in Janskys (column 5), offsets between the optical and IR position are given in arcseconds (column 6), $D$ - distance towards the star (mostly from HIPPARCOS, ESA 1997), and our conclusion about the circumstellar dust presence is given in the last column.

\section{Results and analysis}

24 Be stars with fluxes satisfying the above criteria were found (Table 1). 15 of them have point-like or marginally extended images in at least 3 of 4 IRAS bands, that might be due to radiation of circumstellar dust. 9 sources are either very 
extended or show no concentration on the star's position, and so are probably due to infrared cirrus emission. All the sources are located not far from the zero-age main-sequence (Fig. 1a) and might be Herbig Ae/Be stars, classical Be stars, or proto-planetary nebulae. In particular, HD 38120 was considered a Vega-type star (Coulson, Walther, \& Dent 1998). HD 55439 belongs to the CMa OB1 association and is probably a pre-main-sequence (PMS) star. HD 240010 is in a list of possible PMS objects (Thé, de Winter, \& Pérez 1994).

In order to explain far-IR colors and sizes of the sources a radiation transfer code, DUSTY (Ivezić, Nenkova, \& Elitzur 1999), for spherical dusty envelopes was used. Since the envelopes are optically thin, the exact geometry is not important. The input model parameters were the star's temperature $\left(T_{*}=15000\right.$ $\mathrm{K}$ in all models), dust sublimation temperature $\left(\mathrm{T}_{\mathrm{in}}\right)$, power-law index of the density distribution $(p)$, and overall optical depth (0.01-0.5 in all models). The inner radius of the envelope $\left(r_{1}\right)$ is determined by $\mathrm{T}_{*}$ and $\mathrm{T}_{\mathrm{in}}$, and the outer radius was fixed at $10^{4} r_{1}$. The index $p$ varied from 0 (which corresponds to interstellar dust) to 1.5 (common in PMS stars). Several models were calculated with two power-law density distributions, where $p_{1}=1.5$ is the power-law index between $r_{1}$ and some transition point $Y_{\mathrm{tr}}=300 r_{1}$, and $p_{2}=0$ all the way to the outer radius. Both the IR colors (Fig. 1b) and image sizes of the dusty sources (not shown) are consistent with the model calculations for $p \geq 1$.
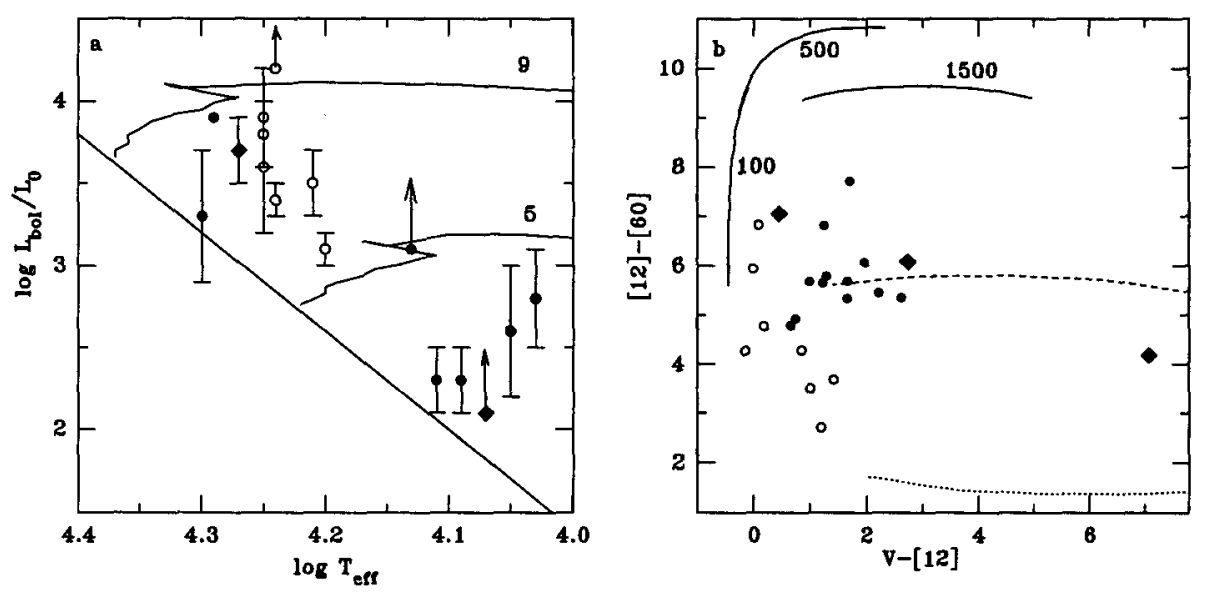

Figure 1. a. The Hertzsprung-Russell diagram. The zero-age mainsequence and evolutionary tracks (marked with initial masses in solar units) from Shaerer et al. (1993) are shown by solid lines. Be stars with dust are denoted by filled circles, those without dust by open circles, PMS candidates by filled diamonds. Error bars are calculated from the distance uncertainty. b. Theoretical color-color tracks and positions of the sample objects (designations are the same as in Fig. 1a). Tracks for $p=0.0$, and different $T_{\text {in }}$ (numbers near the tracks in $K$ ) are shown by solid lines; for $p_{1}=1.5, Y_{\mathrm{tr}}=300 r_{1}, p_{2}=0$ by a dashed line; and for $p=1.5, Y_{\text {out }}=300$ by a dotted line. 


\section{Conclusions}

15 stars with evidence of the presence of circumstellar dust, which is probably remnant of the earlier evolution, are found among galactic Be-type stars. 3 of them are most likely PMS objects (HD 38120, HD 55439, and HD 240010), 9 are classical Be stars or higher-mass counterparts of Vega-type stars, and 3 objects have uncertain classification (HD 251726, HD 147756, and HD 225985). HIRES images are found to be a useful tool for separation of the IR cirrus and circumstellar dust emission that is confirmed by theoretical surface brightness distributions.

\section{References}

Coté, J., Waters, L.B.F.M. 1986, A\&A 176, 93

Coulson, I.M., Walther, D.M., Dent, W.R.F. 1998, MNRAS 296, 934

ESA 1997, The Hipparcos and Tycho Catalogues, ESA SP-1200

Gaustad, J.E., Van Buren, D. 1993, PASP 105, 1127

Ivezić, Ž., Nenkova, M., Elitzur, M. 1999, User Manual for DUSTY, University of Kentucky Internal Report, at http://www.pa.uky.edu/ moshe/dusty

Jaschek, C., Egret, D. 1982, in Be Stars, Proc. IAU Symposium 98, ed. M. Jashek \& H. G. Groth, 261

Miroshnichenko, A.S., et al. 1999, MNRAS 302, 612

Shaerer, D., et al. 1992, A\&AS 102, 339

Thé, P.S., de Winter, D., Pérez, M.R. 1994, A\&AS 104, 315 